\title{
P85 Acute Systemic Inflammation Reduces both Carotid and Aortic Wave Reflection in Young Healthy Adults
}

\author{
Elizabeth Schroeder*, Wesley Lefferts, Thessa Hilgenkamp, Bo Fernhall
}

University of Illinois at Chicago, Chicago, IL, United States

\section{ABSTRACT}

Acute inflammation increases the risk of cardiac and cerebrovascular events, potentially associated with altered hemodynamic load. Wave reflection at the aorta and carotid artery can contribute to central hemodynamic load and provide insight into downstream changes in vascular tone. Acute inflammation may reduce wave reflection via downstream vasodilation, however, this is not firmly established and has only been investigated at the aorta.

Purpose: Explore the effect of acute inflammation on aortic and carotid hemodynamics and wave reflection.

Methods: Twenty-three young adults $\left(26 \pm 4 \mathrm{yrs}, 22.7 \pm 3.2 \mathrm{~kg} / \mathrm{m}^{2}\right)$ underwent vascular measures at baseline and $24 \mathrm{~h}$ following typhoid vaccine-induced inflammation. Aortic (estimated from radial) and carotid pressure waveforms were obtained via tonometry. Augmentation index and wave separation analyses (WSA) were used to assess wave reflection. Aortic stiffness was estimated from single-point pulse wave velocity (PWV). Carotid beta-stiffness and arterial diameters were assessed via ultrasound.

Results: Acute inflammation reduced aortic and carotid wave reflection at $24 \mathrm{~h}$ (See Table $1 \mathrm{AIx}$, Reflection index, $p<0.05$ ). WSA did not reveal independent changes in forward or reflected wave magnitude, time to reflection, PWV, or beta-stiffness $(p>0.05)$. Brachial and carotid artery diameter increased from $3.79 \pm 0.76$ to $3.86 \pm 0.76 \mathrm{~mm}(p=0.055)$ and $6.44 \pm 0.72$ to $6.55 \pm 0.69 \mathrm{~mm}(p=0.04)$, respectively.

Conclusion: Acute inflammation reduces aortic and carotid wave reflection in young adults, potentially due to downstream vasodilation. Although reduced wave reflection can decrease cardiac workload, reduced wave reflection in the carotid may render the brain vulnerable to pulsatile blood flow. These findings may implicate changes in wave reflections are a contributing mechanism behind acute inflammation-induced cerebrovascular risk.

Table 1

\begin{tabular}{|c|c|c|c|c|c|c|c|}
\hline & \multicolumn{4}{|c|}{ Aortic } & \multicolumn{3}{|c|}{ Carotid } \\
\hline & $n$ & Baseline & 24-h & $p$-value & Baseline & 24-h & $p$-value \\
\hline SBP, mmHg & 23 & $104 \pm 10$ & $104 \pm 8$ & 0.88 & $107 \pm 11$ & $107 \pm 10$ & 0.98 \\
\hline DBP, mmHg & 23 & $72 \pm 8$ & $71 \pm 7$ & 0.23 & $71 \pm 8$ & $70 \pm 7$ & 0.26 \\
\hline MAP, mmHg & 23 & $86 \pm 9$ & $85 \pm 7$ & 0.31 & $86 \pm 9$ & $85 \pm 7$ & 0.29 \\
\hline Pulse pressure, $\mathrm{mmHg}$ & 23 & $32 \pm 6$ & $33 \pm 8$ & 0.21 & $36 \pm 7$ & $37 \pm 8$ & 0.39 \\
\hline $\mathrm{AIx}, \%$ & 23 & $3 \pm 10$ & $-1 \pm 10$ & 0.04 & $-4 \pm 15$ & $-9 \pm 16$ & 0.04 \\
\hline AIx@75, \% & 23 & $-6 \pm 12$ & $-9 \pm 12$ & 0.048 & $-13 \pm 15$ & $-18 \pm 15$ & 0.09 \\
\hline Augmented pressure, $\mathrm{mmHg}$ & 23 & $1 \pm 3$ & $0 \pm 3$ & 0.05 & $-2 \pm 6$ & $-4 \pm 7$ & 0.08 \\
\hline $\mathrm{Pf}, \mathrm{mmHg}$ & 22 & $30 \pm 7$ & $31 \pm 7$ & 0.40 & $34 \pm 8$ & $36 \pm 12$ & 0.07 \\
\hline $\mathrm{Pb}, \mathrm{mmHg}$ & 22 & $14 \pm 3$ & $13 \pm 4$ & 0.24 & $16 \pm 3$ & $15 \pm 4$ & 0.09 \\
\hline Reflection index, au & 22 & $47 \pm 8$ & $44 \pm 9$ & 0.04 & $48 \pm 11$ & $43 \pm 13$ & 0.01 \\
\hline Time to reflection, ms & 22 & $158 \pm 16$ & $161 \pm 20$ & 0.20 & $180 \pm 25$ & $185 \pm 26$ & 0.53 \\
\hline
\end{tabular}

All data mean \pm standard deviation. SBP, systolic blood pressure; DBP, diastolic blood pressure; MAP, mean arterial pressure; AIx, augmentation index; Pf, forward wave magnitude; $\mathrm{Pb}$, reflected wave magnitude.

(c) 2019 Association for Research into Arterial Structure and Physiology. Publishing services by Atlantis Press International B.V. This is an open access article distributed under the CC BY-NC 4.0 license (http://creativecommons.org/licenses/by-nc/4.0/). 